\title{
Alterations in white matter volume and integrity in obesity and type 2 diabetes
}

\author{
Liselotte van Bloemendaal ${ }^{1}$ - Richard G. Ijzerman ${ }^{1}$ - Jennifer S. ten Kulve ${ }^{1}$. \\ Frederik Barkhof $^{2} \cdot$ Michaela Diamant $^{1}$ - Dick J. Veltman ${ }^{3} \cdot$ Eelco van Duinkerken $^{1,4,5}$
}

Received: 27 August 2015 / Accepted: 8 January 2016 / Published online: 27 January 2016

(C) The Author(s) 2016. This article is published with open access at Springerlink.com

\begin{abstract}
Type 2 diabetes mellitus (T2DM) is characterized by obesity, hyperglycemia and insulin resistance. Both T2DM and obesity are associated with cerebral complications, including an increased risk of cognitive impairment and dementia, however the underlying mechanisms are largely unknown. In the current study, we aimed to determine the relative contributions of obesity and the presence of T2DM to altered white matter structure. We used diffusion tensor imaging (DTI) and voxel-based morphometry (VBM) to measure white matter integrity and volume in obese T2DM patients without micro- or macrovascular complications, age- genderand BMI-matched normoglycemic obese subjects and ageand gender-matched normoglycemic lean subjects. We found that obese T2DM patients compared with lean subjects had lower axial diffusivity (in the right corticospinal tract, right inferior fronto-occipital tract, right superior longitudinal fasciculus and right forceps major) and reduced white matter volume (in the right inferior parietal lobe and the left external capsule region). In normoglycemic obese compared with lean
\end{abstract}

Liselotte van Bloemendaal

l.vanbloemendaal@vumc.nl

1 Diabetes Center / Department of Internal Medicine, VU University Medical Center, PO BOX 7057, 1007

MB Amsterdam, The Netherlands

2 Department of Radiology \& Nuclear Medicine, VU University Medical Center, 1007 MB Amsterdam, The Netherlands

3 Department of Psychiatry, VU University Medical Center, 1007 MB Amsterdam, The Netherlands

4 Department of Medical Psychology, VU University Medical Center, 1007 MB Amsterdam, The Netherlands

5 Department of Psychology, Pontifícia Universidade Católica (PUC-Rio), Rio de Janeiro, RJ, Brazil subjects axial diffusivity as well as white matter volume tended to be reduced, whereas there were no significant differences between normoglycemic obese subjects and T2DM patients. Decreased white matter integrity and volume were univariately related to higher age, being male, higher BMI, $\mathrm{HbA} 1 \mathrm{C}$ and fasting glucose and insulin levels. However, multivariate analyses demonstrated that only BMI was independently related to white matter integrity, and age, gender and BMI to white matter volume loss. Our data indicate that obese T2DM patients have reduced white matter integrity and volume, but that this is largely explained by BMI, rather than T2DM per se.

Keywords Brain $\cdot$ White matter $\cdot$ Volume $\cdot$ Integrity $\cdot$ Type 2 diabetes $\cdot$ Obesity $\cdot$ DTI $\cdot$ VBM

\section{Introduction}

Obesity and type 2 diabetes mellitus (T2DM) are major public health problems, not only due to their pandemic occurrence, but also due to their association with adverse consequences, such as cardiovascular disease, cancer and cerebral complications (Kullmann et al. 2015; Geijselaers et al. 2015). T2DM is characterized by hyperglycemia and obesity-related insulin resistance (Kahn 2003; Kahn et al. 2006). Patients with T2DM are at an increased risk of (vascular) dementia (Crane et al. 2013), stroke (Aoki and Uchino 2011), white matter lesions (Roriz-Filho et al. 2009) and cognitive impairment (Benedict et al. 2012; Reijmer et al. 2010). Furthermore, T2DM is related to loss of grey and white matter volume (Moran et al. 2013) and to loss of white matter integrity (Reijmer et al. 2010). Although less well established, obesity is also associated with brain disease, including an increased risk of dementia and accelerated cognitive decline at older 
age, with complementary structural brain changes (Gustafson et al. 2003; Gunstad et al. 2007; Kullmann et al. 2015). Both obesity-related insulin resistance and hyperglycemia seem strong risk factors for cerebral pathology (Rusinek and Convit 2014).

Studies assessing brain alterations in T2DM usually include a heterogeneous sample of patients in early and more advanced stages of the disease, i.e., with clinically manifest microvascular and cardiovascular complications. It is therefore not yet clear to what extent structural brain changes are present in early stages of T2DM. Imaging studies in obese subjects have likewise included heterogeneous or inadequately characterized samples with regard to glucose tolerance, insulin resistance, and sometimes cardiovascular disease (Rusinek and Convit 2014).

In the current study, we aimed to determine the relative contributions of obesity and the presence of noncomplicated T2DM to altered white matter volume and integrity. We therefore performed voxel-based morphometry (VBM) and diffusion tensor imaging (DTI) in obese T2DM patients, BMI-matched normoglycemic obese subjects and normoglycemic lean subjects. We hypothesized that white matter integrity and volume are altered in obese T2DM patients compared with BMI-matched normoglycemic obese subjects and lean subjects.

\section{Methods}

\section{Participants}

This study, part of a larger study (NCT01281228), was approved by the Medical Ethics Committee of the VU University Medical Center and was performed in accordance with the Helsinki Declaration. All participants provided written informed consent before participation. We included 16 obese T2DM patients, 16 obese normoglycemic and 16 healthy lean individuals. Inclusion and exclusion criteria have been reported previously (van Bloemendaal et al. 2014). Briefly, inclusion criteria included right-handedness, BMI $>30 \mathrm{~kg} / \mathrm{m}^{2}$ for obese individuals and T2DM patients, BMI $<25 \mathrm{~kg} / \mathrm{m}^{2}$ for lean controls, normoglycemia for obese individuals and lean controls as defined by fasting plasma glucose $<5.6 \mathrm{mmol} / \mathrm{l}$ and 2 -h glucose $<7.8 \mathrm{mmol} / \mathrm{l}$ following a $75 \mathrm{~g}$ oral glucose tolerance test (OGTT). For T2DM patients, $\mathrm{HbA} 1 \mathrm{c}$ had to be $6.0-8.5 \%$. Exclusion criteria included cardiovascular diseases, micro-albuminuria, neurological or psychiatric disorders including depression (assessed by Center for Epidemiologic Studies Depression scale) (Schroevers et al. 2000), substance abuse or use of any centrally acting agent. Microalbuminuria was tested by calculating the albumin: creatinine ratio in urine, and was defined as an albumin:creatinine ratio $>2.5$ for men or $>3.5$ for women.

\section{Data acquisition}

MRI scanning was performed on a 3.0 Tesla GE Signa HDxt scanner (General Electric, Milwaukee, Wisconsin, USA) using an 8-channel phased-array head coil. For this study we used an echo planar imaging based DTI acquisition consisting of 5 volumes without directional weighting and 30 volumes with 30 non-collinear diffusion gradient directions (b-value $1000 \mathrm{~s} / \mathrm{mm}^{2}$, repetition time (TR) $6200 \mathrm{~ms}$, echo time (TE) $81 \mathrm{~ms}, 45$ contiguous axial slices of $2.4 \mathrm{~mm}$ ). Participants furthermore had a T2-based Fluid Attenuating Inverse Recovery (3D-FLAIR; TR $8000 \mathrm{~ms}$, TE $126 \mathrm{~ms}$, slice thickness $1.2 \mathrm{~mm}$ ) and a T1-weighted fast spoiled gradient-echo (TR $8.2 \mathrm{~ms}$, TE $3.2 \mathrm{~ms}, 1 \mathrm{~mm}$ slice thickness) sequence.

\section{DTI tract-based analysis}

Processing of DTI-scans was performed using the FMRIB's Diffusion Toolbox of FMRI's Software Library (FSL) version 5.04 (http://fsl.fmrib.ox.ac.uk/fsl/fslwiki) (Smith et al. 2004). First, DTI scans and gradient-vectors were corrected for motion and eddy-current induced distortions (Leemans and Jones 2009). Next, the diffusion tensor was calculated for each voxel, providing fractional anisotropy (FA), axial $(\lambda 1)$, radial (mean of $\lambda 2$ and $\lambda 3$ ) and mean diffusivity (mean of $\lambda 1, \lambda 2$ and $\lambda 3$ ) (Pierpaoli and Basser 1996; Basser and Jones 2002). Tract-based spatial statistics (TBSS) was used for voxelwise statistical analysis, which does not use spatial smoothing (Smith et al. 2006). All individual FA images were nonlinearly registered to FMRIB58FA standard space, to allow group averaging and comparison. These registered images were then averaged and the mean image was skeletonized, and thresholded at 0.2 to include only white matter. Individual non-linear warps and skeleton projection of FA-images were used to project axial, radial and mean diffusivity to the skeleton and to allow voxelwise statistics. All steps were manually checked, no errors occurred.

\section{VBM analysis}

T1-weighted images were visually inspected for motion artefacts. Data preprocessing was performed with Statistical Parametric Mapping 8 software (SPM8; Wellcome Trust Center for Neuroimaging, London, UK; http://www.fil.ion. ucl.ac.uk/spm). First, structural images were oriented along the anterior/posterior commissure axis and segmented into grey matter, white matter and cerebrospinal fluid with a biasfield correction cut-off of $60 \mathrm{~mm}$ full width at half maximum (FWHM). In the next step, DARTEL (Diffeomorphic Anatomical Registration Through Exponentiated Lie Algebra) was used to create a group-specific grey and white matter template and the individual flow fields. These flow fields contain the non-linear deformations between each 
individual's MRI-scan and the DARTEL template. The individual grey and white matter segmentations were registered to MNI standard space using linear affine registration and nonlinear deformation using the flow fields. The images were modulated to preserve relative volume and corrected for brain size, in order to allow group comparisons. Lastly, the segmented, modulated and normalized images were smoothed using an $8 \mathrm{~mm}$ FWHM Gaussian kernel. Both segmentation and normalization results were manually checked, no errors occurred.

\section{White matter lesions}

White matter lesions were scored visually by an experienced neuro-radiologist (FB) based on 3D-FLAIR sequence using Fazekas criteria (Fazekas et al. 1987).

\section{Statistical analyses}

Clinical group data are expressed as mean \pm SEM (unless otherwise stated) and were analyzed with the Statistical Package for the Social Sciences (SPSS) version 20 (SPSS, Chicago, IL, USA). Between group differences in clinical data, including white matter lesions, were analyzed using a oneway ANOVA with Bonferroni post-hoc correction or $\chi^{2}$-test whether appropriate.

Differences in DTI parameters between groups were analyzed with the FSL function "randomise", using thresholdfree cluster enhancement non-parametric permutation testing (5000 permutations). This method does not require a minimum cluster size to be defined a priori. Family Wise Error (FWE) was applied as multiple comparisons correction. For the VBM analysis of white matter volume, clusters were considered relevant if at least 150 voxels at $\mathrm{P}_{\text {uncorrected }}<0.002$. Those clusters were subsequently whole brain cluster-wise corrected for multiple comparisons using FWE. This minimum of 150 voxels ensured that only larger, more relevant alteration patterns were found, reducing the risk of falsepositive findings and increasing the reliability of the results.

Both the DTI and VBM analyses were corrected for age, gender and systolic blood pressure. A $P$-value $<0.05$ after FWE-correction was considered statistically significant.

Using FSL the mean value of white matter integrity of voxels differing between groups was extracted. For each subject the mean white matter volume of significant clusters was extracted using MarsBar 0.41 (http://marsbar.sourceforge. net). Using Spearman's $\rho$ the demographical and clinical correlates of altered volume/integrity were determined. These correlations were calculated in all participants. To determine the strongest predictors, variables significantly correlating with volume/integrity, were entered in a forward regression model.

\section{Results}

\section{Group demographics}

Due to technical problems, DTI scans were not available for 1 lean control and 1 obese subject. All subjects were matched for age and gender, while T2DM patients and normoglycemic obese subjects were also BMI matched (Table 1). Patients with T2DM had higher systolic blood pressure, HbA1c, fasting plasma glucose and insulin levels compared with normoglycemic obese and lean subjects. Total cholesterol and LDL cholesterol levels were lower in T2DM patients compared with obese and lean subjects, whereas HDL cholesterol levels were higher in lean compared with T2DM and obese subjects. Eight T2DM patients were treated with metformin monotherapy and 8 used metformin in combination with a sulfonylurea. Twelve T2DM patients and 3 obese subjects used antihypertensive medication, 13 T2DM patients and 1 obese subject used statins. There were no significant differences in Fazekas score (white matter lesions) between the groups $(P=0.8)$ (Table 1$)$.

\section{White matter integrity}

DTI tract-based analyses showed significantly lower axial diffusivity $\left(\lambda_{1}\right)$ in T2DM patients compared with lean subjects. Tracts most affected were the right corticospinal tract, right inferior fronto-occipital tract, right superior longitudinal fasciculus and right forceps major (Fig. 1a). On the left side of the forceps major there was a cluster of voxels where axial diffusivity tended to be lower in subjects with obesity compared with lean controls $\left(\mathrm{P}_{\mathrm{FWE}}=0.1\right.$; Fig. 1b). There were no significant differences in FA, mean diffusivity and radial diffusivity between T2DM patients, normoglycemic obese and lean subjects.

\section{White matter volume}

VBM analyses demonstrated significantly lower white matter volume in T2DM patients relative to lean control subjects in a left hemisphere cluster comprising predominantly the external capsule region. Another cluster of lower white matter volume in T2DM patients versus lean control subjects was found in the right inferior parietal lobe (Fig. 2a; Table 2).

In normoglycemic obese compared with lean subjects, lower white matter volume was found in the left external capsule region as well (Fig. 2b; Table 2), although this cluster was smaller than the cluster found in T2DM patients. In addition, this cluster was not statistically significant after FWEcorrection for multiple comparisons $\left(\mathrm{P}_{\mathrm{FWE}}=0.380\right)$. No differences were identified in the comparison between normoglycemic obese subjects and T2DM patients. 
Table 1 Subject characteristics

\begin{tabular}{lllll}
\hline & Lean $(n=15)$ & Obese $(n=15)$ & T2DM $(n=16)$ & ANOVA $P$-value \\
\hline Age (years) & $57.3 \pm 1.9$ & $57.7 \pm 2.2$ & $61.4 \pm 1.5$ & 0.2 \\
Gender, male/female (n) & $8 / 7$ & $8 / 7$ & $8 / 8$ & - \\
Weight (kg) & $71.5 \pm 2.9$ & $100.7 \pm 3.0^{*}$ & $97.9 \pm 3.0^{*}$ & $<0.001$ \\
Body mass index (kg/m $\left.{ }^{2}\right)$ & $23.4 \pm 0.4$ & $32.6 \pm 0.8^{*}$ & $34.0 \pm 0.9^{*}$ & $<0.001$ \\
Waist circumference (cm) & $85.7 \pm 2.1$ & $112.3 \pm 2.2^{*}$ & $115.7 \pm 1.8^{*}$ & $<0.001$ \\
Systolic blood pressure (mmHg) & $119 \pm 4$ & $127 \pm 3$ & $141 \pm 3 \dagger$ & $<0.001$ \\
Diastolic blood pressure (mmHg) & $76 \pm 2$ & $79 \pm 2$ & $83 \pm 2$ & 0.057 \\
Fasting plasma glucose (mmol/l) & $5.2 \pm 0.1$ & $5.3 \pm 0.1$ & $8.4 \pm 0.5 \dagger$ & $<0.001$ \\
Glucose 2 h after OGTT (mmol/l) & $5.1 \pm 0.3$ & $5.4 \pm 0.2$ & - & 0.4 \\
HbA1c (\%) & $5.5 \pm 0.03$ & $5.5 \pm 0.06$ & $6.9 \pm 0.22 \dagger$ & $<0.001$ \\
HbA1c (mmol/mol) & $37 \pm 0.3$ & $37 \pm 0.7$ & $52 \pm 2.4 \dagger$ & $<0.001$ \\
Total cholesterol (mmol/l) & $5.6 \pm 0.2$ & $5.6 \pm 0.2$ & $4.5 \pm 0.3 \dagger$ & 0.002 \\
LDL-cholesterol (mmol/1) & $3.3 \pm 0.2$ & $3.4 \pm 0.2$ & $2.3 \pm 0.2 \dagger$ & $<0.001$ \\
HDL-cholesterol (mmol/l) & $1.9 \pm 0.1$ & $1.4 \pm 0.1^{*}$ & $1.3 \pm 0.1 *$ & $<0.001$ \\
Triglycerids (mmol/l) & $0.9 \pm 0.1$ & $1.7 \pm 0.3$ & $1.8 \pm 0.3$ & 0.046 \\
Fasting NEFA (mmol/l) & $0.46 \pm 0.04$ & $0.45 \pm 0.03$ & $0.64 \pm 0.04 \dagger$ & 0.001 \\
Fasting insulin (pmol/l) & $36 \pm 2.8$ & $81 \pm 14$ & $117 \pm 17 \dagger$ & $<0.001$ \\
Diabetes duration (years) & - & - & $7.0[4.25,10.75]$ & - \\
Fazekas score (0; 1; 2; 3) & $6 ; 7 ; 2 ; 0$ & $8 ; 5 ; 2 ; 0$ & $5 ; 9 ; 2 ; 0$ & 0.8 \\
\hline
\end{tabular}

Data are means \pm SEM or median [interquartile range]

Fazekas score for white matter lesions: 0 indicates no lesions; 1 indicates punctate foci; 2 indicates beginning confluence of foci; 3 indicates large confluent areas

OGTT oral glucose tolerance test, NEFA non-esterified fatty acids, T2DM type 2 diabetes patients

* Statistically significant different from lean (post-hoc Bonferroni corrected $P<0.05$ )

$\dagger$ Statistically significant different from lean and obese (post-hoc Bonferroni corrected $P<0.05$ )

\section{Associations between white matter parameters and biomedical variables}

For each subject the mean axial diffusivity value was extracted for the significant voxels in the comparison between T2DM patients and lean controls. The same was done for white matter volume of the two VBM clusters that reached statistical significance when comparing T2DM patients with lean control subjects. With these values correlations with demographic and clinical characteristics were calculated in the overall group. As can be found in Table 3, univariate correlations of lower axial diffusivity with higher BMI, fasting plasma glucose, fasting insulin levels and HbAlc were significant. Of these variables, higher BMI was the only independent predictor of lower axial diffusivity $(\beta=-0.48 ; P=0.001)$, which explained $21 \%$ of the variance. Lower white matter volume in the left external capsule cluster was univariately related to higher age, BMI, fasting plasma glucose and insulin levels, HbAlc, and being male. Being male $(\beta=0.432 ; P<0.001)$, higher BMI $(\beta=-0.353 ; P=0.002)$ and higher age $(\beta=-0.342 ; P=0.003)$ were independently related with lower white matter volume in this cluster. This model explained $47 \%$ of the variance of white matter volume differences in this cluster. Similar univariate correlations were found for the cluster of lower white matter volume in the right inferior parietal lobe, with the exception of fasting plasma glucose (Table 2). Independent predictors were gender (being male; $\beta=0.449 ; P<0.001)$, higher age $(\beta=-0.345 ; P=0.004)$, and higher BMI $(\beta=-0.284 ; P=0.016)$, together explaining $44 \%$ of the variance.

\section{Discussion}

In the current study we showed that both white matter integrity, as measured by axial diffusivity, and white matter volume are decreased in obese T2DM patients compared with lean subjects. In normoglycemic obese compared with lean subjects axial diffusivity as well as white matter volume tended to be reduced, whereas there were no significant differences between normoglycemic obese subjects and T2DM patients. Higher BMI independently predicted decreased white matter integrity, and, together with higher age and being male, it predicted lower white matter volume as well. Higher $\mathrm{HbA} 1 \mathrm{C}$, fasting plasma glucose and insulin levels were no independent predictors of decreased white matter volume and integrity. 


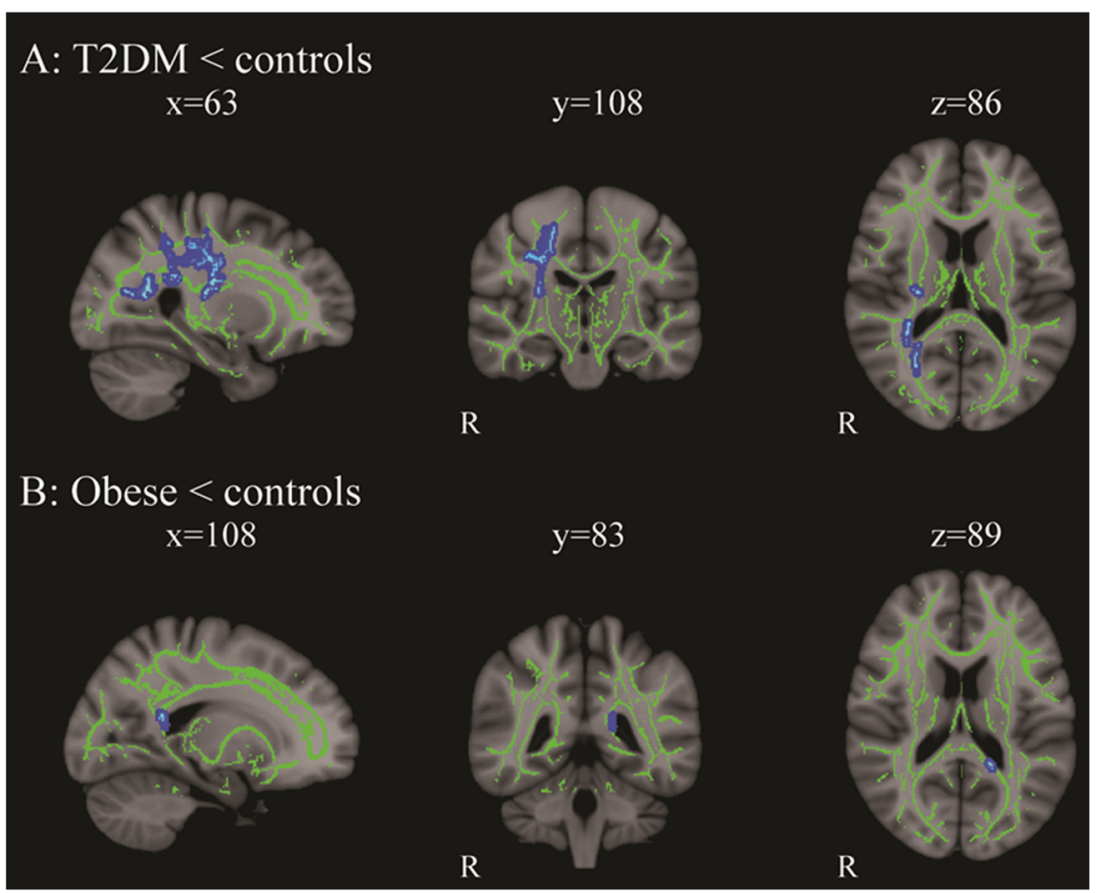

Fig. 1 Changes in axial diffusivity in T2DM patients and obese compared with lean subjects. a Brain slices showing decreased axial diffusivity $\left(\lambda_{1}\right)$ in T2DM patients compared with lean subjects in right corticospinal tract, right inferior fronto-occipital tract, right superior longitudinal fasciculus and right forceps major; $\mathbf{b}$ Brain slices showing a cluster of voxels on the left side of the forceps major where axial

Using DTI, we found that obese T2DM patients compared with lean subjects have lower axial diffusivity in the right corticospinal tract, right inferior fronto-occipital tract, right superior longitudinal fasciculus and right forceps major. Our

\section{A: T2DM $<$ controls}
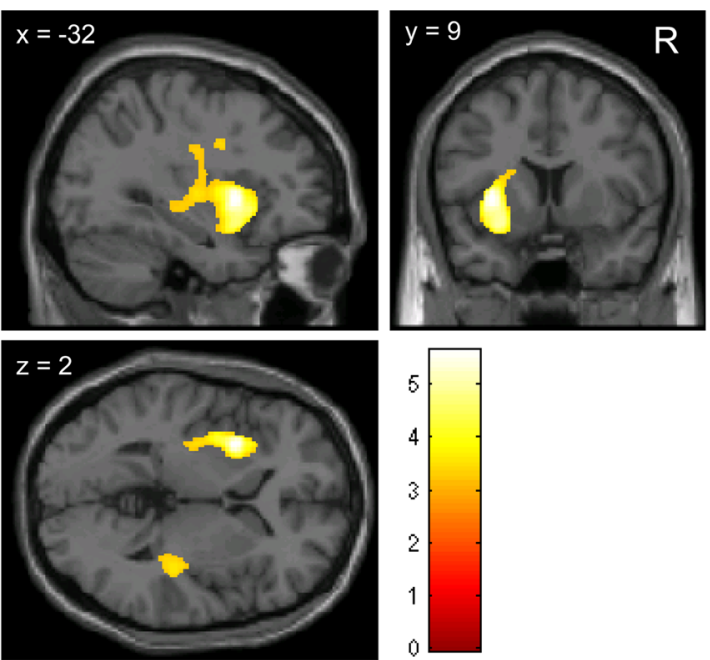

Fig. 2 Reduced white matter volume in T2DM patients compared with lean subjects. a Brain slices showing clusters of reduced white matter volume in the external capsule region in obese T2DM patients compared with lean subjects, as determined with VBM; b Brain slices showing a cluster of reduced white matter volume in the external capsule diffusivity tended to be lower in subjects with obesity compared with controls $\left(\mathrm{P}_{\mathrm{FWE}}=0.11\right)$. The mean skeleton is shown in green, and significant differences are displayed as thickened tracts in blue for visualization purposes. Left side of the axial slices is the right side of the brain. X, y, z are the Montreal Neurological Institute (MNI) coordinates of the brain in standard space

findings are in line with previous studies showing lower axial diffusivity in type 1 diabetes patients with and without microvascular complications in comparable tracts (van Duinkerken et al. 2012) and in T2DM patients (Hoogenboom et al. 2014),

\section{B: Obese < controls}
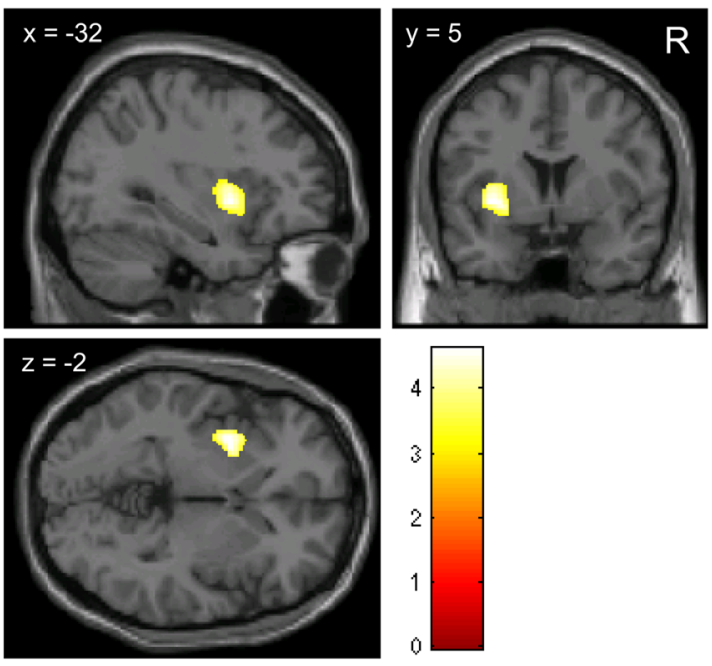

region in obese compared with lean subjects. This cluster, however, was not statistically significant after FWE-correction for multiple comparisons $\left(\mathrm{P}_{\mathrm{FWE}}=0.380\right)$. The color scale reflects the T-value. Right side of the axial slices is the right side of the brain. X, $\mathrm{y}, \mathrm{z}$ are the Montreal Neurological Institute (MNI) coordinates of the brain in standard space 
Table 2 Between-group differences in white matter volume and integrity

\begin{tabular}{|c|c|c|c|c|c|c|c|c|}
\hline & Region & Side & $\begin{array}{l}\text { Cluster } \\
\text { (voxels) }\end{array}$ & $\begin{array}{l}\text { Volume } \\
(\mathrm{mL})\end{array}$ & $\mathrm{T}$ & $\begin{array}{l}\text { P- } \\
\text { uncorr }\end{array}$ & $\mathrm{P}_{-\mathrm{FWE}}$ & $\begin{array}{l}\text { MNI } \\
\text { coordinates } \\
(\mathrm{x}, \mathrm{y}, \mathrm{z})\end{array}$ \\
\hline \multicolumn{9}{|c|}{ White matter volume } \\
\hline \multirow[t]{6}{*}{ T2DM $<$ Lean } & \multirow[t]{3}{*}{ External capsule } & \multirow[t]{3}{*}{$\mathrm{L}$} & \multirow[t]{3}{*}{5735} & \multirow[t]{3}{*}{19.36} & 5.64 & $<0.001$ & $<0.001$ & $-32,9,2$ \\
\hline & & & & & 4.99 & $<0.001$ & $<0.001$ & $-27,8,-13$ \\
\hline & & & & & 4.03 & $<0.001$ & $<0.001$ & $17,-16,33$ \\
\hline & \multirow{3}{*}{$\begin{array}{l}\text { Inferior Parietal } \\
\text { Lobe }\end{array}$} & \multirow[t]{3}{*}{$\mathrm{R}$} & \multirow[t]{3}{*}{3276} & \multirow[t]{3}{*}{11.06} & 4.69 & $<0.001$ & $<0.001$ & $45,-42,54$ \\
\hline & & & & & 4.49 & $<0.001$ & $<0.001$ & $47,-31,30$ \\
\hline & & & & & 4.48 & $<0.001$ & $<0.001$ & $39,-12,36$ \\
\hline Obese $<$ Lean & External capsule & $\mathrm{L}$ & 903 & 3.05 & 4.62 & $<0.001$ & 0.380 & $-32,5,-1$ \\
\hline \multicolumn{9}{|l|}{ Axial diffusivity } \\
\hline T2DM $<$ Lean & $\begin{array}{l}\text { Corticospinal tract, inferior } \\
\text { fronto-occipital tract, } \\
\text { superior } \\
\text { longitudinal fasciculus } \\
\text { and forceps major }\end{array}$ & $\mathrm{R}$ & 1803 & 1.80 & 5.49 & NA & $<0.05$ & $22,-28,46$ \\
\hline
\end{tabular}

$T$ t-statistic, $P_{-F W E} p$-value Family-Wise Error corrected for multiple comparisons, $R$ right, $L$ left, $M N I$ Montreal Neurological Institute coordinates in mm $N A$ not applicable

although the latter study only observed a trendwise reduced axial diffusivity in the cingulum bundle. In the current study, we combined DTI with VBM of white matter and found that T2DM patients have reduced white matter volume in the right inferior parietal lobe and the left external capsule region. A previous study in a large cohort of T2DM patients (of which $24 \%$ had known cardiovascular and $11 \%$ cerebrovascular complications), also demonstrated white matter loss in T2DM, but mainly in frontal and temporal regions (Moran et al. 2013). In addition, in obese adolescents with T2DM versus BMI-matched non-diabetic subjects, reduced frontal and whole brain white matter volume has been demonstrated, paralleled by reductions in cognitive performance (Yau et al. 2010).

To determine the relative contributions of obesity and T2DM to structural brain changes, we also studied normoglycemic obese subjects (BMI-matched with the T2DM patients). We found in normoglycemic obese compared with lean subjects reduced axial diffusivity, as well as reduced white matter volume, but these differences in white matter integrity and volume were only trendwise significant after strict FWE-correction for multiple comparisons. It could be speculated that obesity is associated with early white matter alterations and that hyperglycemia may further impact brain
Table 3 Associations between white matter parameters and demographic and clinical characteristics

\begin{tabular}{|c|c|c|c|c|c|c|}
\hline & \multicolumn{2}{|l|}{ Axial diffusivity ${ }^{a}$} & \multicolumn{2}{|c|}{$\begin{array}{l}\text { White matter volume cluster } \\
\mathrm{L}^{\mathrm{b}}\end{array}$} & \multicolumn{2}{|c|}{$\begin{array}{l}\text { White matter volume cluster } \\
\mathrm{R}^{\mathrm{b}}\end{array}$} \\
\hline & Spearman's rho & $P$-value & Spearman's rho & $P$-value & Spearman's rho & $P$-value \\
\hline Age & -0.09 & 0.573 & -0.34 & 0.021 & -0.30 & 0.041 \\
\hline Gender & 0.27 & 0.069 & 0.52 & $<0.001$ & 0.54 & $<0.001$ \\
\hline BMI & -0.47 & 0.001 & -0.55 & $<0.001$ & -0.43 & 0.003 \\
\hline Fasting glucose & -0.45 & 0.002 & -0.31 & 0.035 & -0.24 & 0.10 \\
\hline Fasting insulin & -0.44 & 0.002 & -0.40 & 0.006 & -0.30 & 0.040 \\
\hline $\mathrm{HbAlc}$ & -0.38 & 0.008 & -0.42 & 0.004 & -0.38 & 0.010 \\
\hline HDL & 0.27 & 0.07 & 0.17 & 0.3 & 0.15 & 0.35 \\
\hline
\end{tabular}

${ }^{a}$ Mean axial diffusivity value for the significant voxels in the comparison between T2DM patients and lean controls

${ }^{\mathrm{b}}$ Mean white matter volume of the two significant VBM clusters in the comparison between T2DM patients and lean controls. With these values correlations with biomedical variables were calculated in all subjects (lean, obese and $\mathrm{T} 2 \mathrm{DM}$ ) 
white matter structure. However, in the current study we found no significant differences between obese T2DM patients and obese normoglycemic subjects in white matter structure.

Relating our findings of localized lower white matter volume and integrity to specific functions is not straightforward given the complexity of brain networks. However, it is well known that the corticospinal tracts have a major role in motor coordination, whereas the forceps major and parietal cortices have been related to the transfer and processing of somatosensory information. In a previous study in T2DM patients white matter integrity of the inferior longitudinal fasciculus has been related to cognitive functioning, mainly information processing speed (Reijmer et al. 2013). Similar correlations between the inferior fronto-occipital tract and cognition were found in type 1 diabetes patients (van Duinkerken et al. 2012). Our finding of reduced white matter volume in the external capsule region in obese T2DM patients furthermore corroborates previous findings of white matter alterations in the external capsule in obese versus lean women (Shott et al. 2015). The external capsule connects medial and ventral prefrontal cortices with limbic regions, contains fibers from both the inferior fronto-occipital fasciculus and uncinate fasciculus, and connects the hippocampus and amygdala with prefrontal and OFC regions (Shott et al. 2015; Schmahmann et al. 2008). Future studies are needed to better understand the consequences of the observed changes in white matter volume and integrity in our study.

We did not observe any differences in FA or any of the other diffusion tensor parameters. Previous studies have shown alterations in FA or mean and radial diffusivity in T2DM (Hoogenboom et al. 2014; Hsu et al. 2010; Reijmer et al. 2013). In the current study we only observed reduced axial diffusivity in T2DM patients. The biological substrate of axial diffusivity has mainly been derived from animal studies, and therefore careful interpretation is required. These animal studies, however, suggest that changes in axial diffusivity may represent changes in integrity of axons (Madden et al. 2009; Wheeler-Kingshott and Cercignani 2009). This could result from a less favorable alignment of fibers within the bundle, but it could also represent damage to the axons themselves. In a mouse model of multiple sclerosis it was demonstrated that greater decreases in axial diffusivity were associated with greater amounts of axonal damage and with more neurological disability (Budde et al. 2008). Further studies are needed to determine the clinical relevance of loss of axial diffusivity in T2DM patients.

Results from the white matter volume analysis appear to be more robust and extensive than those of the white matter integrity analysis. This may seem counterintuitive as white matter integrity is considered to be more sensitive to early alterations than white matter volume. The difference, in this case, may be due to our statistical approach. For the white matter volume analysis, first clusters with a $P<0.002$ and a minimum size of 150 voxels were identified. Those clusters were subsequently whole brain cluster-wise corrected for multiple comparisons using FWE. For the white matter integrity analysis this approach is not available and a whole-brain voxel-wise FWE-correction was used. Obviously, the latter approach is more stringent and will yield less statistically significant voxels. We chose the cluster-wise approach for the volumetric analysis given the small sample size. These different approaches need to be taken into account when comparing both results.

In the overall group we found univariate correlations between altered white matter integrity and volume, age, being male, BMI, and fasting glucose and insulin levels. These correlations suggest that hyperglycemia and insulin-resistance partly relate to obesity/T2DM related cerebral alterations. However, in a multivariate model only BMI was related to white matter integrity, and age, gender and BMI to white matter volume loss. The association between higher BMI and lower white matter volume is in line with a previous study in elderly subjects (Raji et al. 2010). Obesity is characterized by a chronic proinflammatory state (Johnson et al. 2012) and low-grade inflammatory markers have been shown to be related to white matter integrity and other brain parameters (Verstynen et al. 2013; Frodl and Amico 2014). In this study no peripheral or central proinflammatory markers were available, but future research should investigate this issue.

We also determined correlations between white matter integrity/volume and HDL and LDL cholesterol. In the overall group, the mean value of the cluster of lower axial diffusivity, but not white matter volume, tended to correlate positively with both cholesterol types. While the association with HDL cholesterol is as anticipated, the direction of the correlation with LDL seems counterintuitive (higher LDL correlates with higher axial diffusivity values). This finding may however be explained by the statin use in the obese and T2DM group, which lowers LDL cholesterol levels. Therefore, this correlation is likely to be unreliable in our current study design. Ideally, such a correlation should be calculated in individuals not using statins, which was not feasible in the current study due to power issues. We have therefore excluded LDL from the analyses.

For exploratory purposes we have added, besides BMI, other biomedical variables, such as fasting insulin and glucose, to a second level statistical design to test whether regional white matter integrity/volume is related to these variables. However, given the study design, focusing on groups differing with regard to T2DM and BMI, these tests will not be fully independent of BMI. This is a different approach than the approach we took by correlating clusters of altered integrity/volume to biomedical variables as a post-hoc analysis. This second level whole brain statistical analysis did not show any statistically significant correlations between white matter integrity/volume and fasting plasma glucose or fasting plasma insulin. 
A strength of this study is that groups were wellphenotyped as we only included patients with noncomplicated T2DM, BMI-matched normoglycemic subjects and healthy lean subjects. A limitation of our study is the relatively small sample size, limiting the power to detect significant differences between the groups. However, despite the small sample size we observed significant differences between T2DM patients and lean subjects in measures of white matter tract integrity and white matter volume. Another limitation of the current study is that measurements of cognitive functions were not performed. Previous studies have demonstrated associations between reduced white matter volume/integrity and cognitive functions in T2DM as well as in obesity (Hoogenboom et al. 2014; Moran et al. 2013; Reijmer et al. 2013; Zhang et al. 2014; Kullmann et al. 2015). Reduced white matter integrity and volume may play a key role in obesity- and T2DM-related cognitive impairment (Kullmann et al. 2015).

In conclusion, we found that both white matter integrity and white matter volume are focally decreased in obese patients with non-complicated T2DM compared with lean subjects. In normoglycemic obese compared with lean subjects axial diffusivity as well as white matter volume only tended to be reduced. Higher BMI was an independent predictor of decreased white matter integrity as well as white matter volume. Our data indicate that obese T2DM patients have reduced white matter integrity and volume, but that this is largely explained by BMI, rather than the presence of T2DM per se.

\section{Compliance with ethical standards}

Funding This research was supported by an investigator-initiated grant from Eli Lilly and Company and Bristol-Myers Squibb (BMS). Dr. IJzerman is financed by the Netherlands Organisation for Scientific Research (NWO) Innovational Research Incentives Scheme Veni (no. 91613082).

Open Access This article is distributed under the terms of the Creative Commons Attribution 4.0 International License (http:// creativecommons.org/licenses/by/4.0/), which permits unrestricted use, distribution, and reproduction in any medium, provided you give appropriate credit to the original author(s) and the source, provide a link to the Creative Commons license, and indicate if changes were made.

\section{References}

Aoki J, Uchino K (2011) Treatment of risk factors to prevent stroke. Neurotherapeutics 8:463-474

Basser PJ, Jones DK (2002) Diffusion-tensor MRI: theory, experimental design and data analysis - a technical review. NMR Biomed 15:456467

Benedict C, Brooks SJ, Kullberg J, Burgos J, Kempton MJ, Nordenskjold R, Nylander R, Kilander L, Craft S, Larsson EM, Johansson L, Ahlstrom H, Lind L, Schioth HB (2012) Impaired insulin sensitivity as indexed by the HOMA score is associated with deficits in verbal fluency and temporal lobe gray matter volume in the elderly. Diabetes Care 35:488-494

Budde MD, Kim JH, Liang HF, Russell JH, Cross AH, Song SK (2008) Axonal injury detected by in vivo diffusion tensor imaging correlates with neurological disability in a mouse model of multiple sclerosis. NMR Biomed 21:589-597

Crane PK, Walker R, Hubbard RA, Li G, Nathan DM, Zheng H, Haneuse S, Craft S, Montine TJ, Kahn SE, McCormick W, McCurry SM, Bowen JD, Larson EB (2013) Glucose levels and risk of dementia. N Engl J Med 369:540-548

Fazekas F, Chawluk JB, Alavi A, Hurtig HI, Zimmerman RA (1987) MR signal abnormalities at $1.5 \mathrm{~T}$ in Alzheimer's dementia and normal aging. AJR Am J Roentgenol 149:351-356

Frodl T, Amico F (2014) Is there an association between peripheral immune markers and structural/functional neuroimaging findings? Prog Neuropsychopharmacol Biol Psychiatry 48:295-303

Geijselaers SL, Sep SJ, Stehouwer CD, Biessels GJ (2015) Glucose regulation, cognition, and brain MRI in type 2 diabetes: a systematic review. Lancet Diabetes Endocrinol 3:75-89

Gunstad J, Paul RH, Cohen RA, Tate DF, Spitznagel MB, Gordon E (2007) Elevated body mass index is associated with executive dysfunction in otherwise healthy adults. Compr Psychiatry 48:57-61

Gustafson D, Rothenberg E, Blennow K, Steen B, Skoog I (2003) An 18year follow-up of overweight and risk of Alzheimer disease. Arch Intern Med 163:1524-1528

Hoogenboom WS, Marder TJ, Flores VL, Huisman S, Eaton HP, Schneiderman JS, Bolo NR, Simonson DC, Jacobson AM, Kubicki M, Shenton ME, Musen G (2014) Cerebral white matter integrity and resting-state functional connectivity in middle-aged patients with type 2 diabetes. Diabetes 63:728-738

Hsu JL, Van HW, Bai CH, Lee CH, Tsai YF, Chiu HC, Jaw FS, Hsu CY, Leu JG, Chen WH, Leemans A (2010) Microstructural white matter changes in normal aging: a diffusion tensor imaging study with higher-order polynomial regression models. Neuroimage 49:32-43

Johnson AR, Milner JJ, Makowski L (2012) The inflammation highway: metabolism accelerates inflammatory traffic in obesity. Immunol Rev 249:218-238

Kahn SE (2003) The relative contributions of insulin resistance and betacell dysfunction to the pathophysiology of Type 2 diabetes. Diabetologia 46:3-19

Kahn SE, Hull RL, Utzschneider KM (2006) Mechanisms linking obesity to insulin resistance and type 2 diabetes. Nature 444:840-846

Kullmann S, Schweizer F, Veit R, Fritsche A, Preissl H (2015) Compromised white matter integrity in obesity. Obes Rev 16:273281

Leemans A, Jones DK (2009) The B-matrix must be rotated when correcting for subject motion in DTI data. Magn Reson Med 61: 1336-1349

Madden DJ, Bennett IJ, Song AW (2009) Cerebral white matter integrity and cognitive aging: contributions from diffusion tensor imaging. Neuropsychol Rev 19:415-435

Moran C, Phan TG, Chen J, Blizzard L, Beare R, Venn A, Munch G, Wood AG, Forbes J, Greenaway TM, Pearson S, Srikanth V (2013) Brain atrophy in type 2 diabetes: regional distribution and influence on cognition. Diabetes Care 36:4036-4042

Pierpaoli C, Basser PJ (1996) Toward a quantitative assessment of diffusion anisotropy. Magn Reson Med 36:893-906

Raji CA, Ho AJ, Parikshak NN, Becker JT, Lopez OL, Kuller LH, Hua X, Leow AD, Toga AW, Thompson PM (2010) Brain structure and obesity. Hum Brain Mapp 31:353-364

Reijmer YD, Brundel M, de Bresser J, Kappelle LJ, Leemans A, Biessels GJ (2013) Microstructural white matter abnormalities and cognitive functioning in type 2 diabetes: a diffusion tensor imaging study. Diabetes Care 36:137-144 
Reijmer YD, van den Berg E, Ruis C, Kappelle LJ, Biessels GJ (2010) Cognitive dysfunction in patients with type 2 diabetes. Diabetes Metab Res Rev 26:507-519

Roriz-Filho S, Sa-Roriz TM, Rosset I, Camozzato AL, Santos AC, Chaves ML, Moriguti JC, Roriz-Cruz M (2009) (Pre)diabetes, brain aging, and cognition. Biochim Biophys Acta 1792:432-443

Rusinek H, Convit A (2014) Obesity: cerebral damage in obesityassociated metabolic syndrome. Nat Rev Endocrinol 10:642-644

Schmahmann JD, Smith EE, Eichler FS, Filley CM (2008) Cerebral white matter: neuroanatomy, clinical neurology, and neurobehavioral correlates. Ann N Y Acad Sci 1142:266-309

Schroevers MJ, Sanderman R, van SE, Ranchor AV (2000) The evaluation of the Center for Epidemiologic Studies Depression (CES-D) scale: depressed and positive affect in cancer patients and healthy reference subjects. Qual Life Res 9:1015-1029

Shott ME, Cornier MA, Mittal VA, Pryor TL, Orr JM, Brown MS, Frank GK (2015) Orbitofrontal cortex volume and brain reward response in obesity. Int J Obes (Lond) 39:214-221

Smith SM, Jenkinson M, Johansen-Berg H, Rueckert D, Nichols TE, Mackay CE, Watkins KE, Ciccarelli O, Cader MZ, Matthews PM, Behrens TE (2006) Tract-based spatial statistics: voxelwise analysis of multi-subject diffusion data. Neuroimage 31:1487-1505

Smith SM, Jenkinson M, Woolrich MW, Beckmann CF, Behrens TE, Johansen-Berg H, Bannister PR, De LM, Drobnjak I, Flitney DE,
Niazy RK, Saunders J, Vickers J, Zhang Y, De SN, Brady JM, Matthews PM (2004) Advances in functional and structural MR image analysis and implementation as FSL. Neuroimage 23 (Suppl 1):S208-S219

van Bloemendaal L, Ijzerman RG, Ten Kulve JS, Barkhof F, Konrad RJ, Drent ML, Veltman DJ, Diamant M (2014) GLP-1 receptor activation modulates appetite- and reward-related brain areas in humans. Diabetes 63:4186-4196

van Duinkerken E, Schoonheim MM, Ijzerman RG, Klein M, Ryan CM, Moll AC, Snoek FJ, Barkhof F, Diamant M, Pouwels PJ (2012) Diffusion tensor imaging in type 1 diabetes: decreased white matter integrity relates to cognitive functions. Diabetologia 55:1218-1220

Verstynen TD, Weinstein A, Erickson KI, Sheu LK, Marsland AL, Gianaros PJ (2013) Competing physiological pathways link individual differences in weight and abdominal adiposity to white matter microstructure. Neuroimage 79:129-137

Wheeler-Kingshott CA, Cercignani M (2009) About "axial" and "radial" diffusivities. Magn Reson Med 61:1255-1260

Yau PL, Javier DC, Ryan CM, Tsui WH, Ardekani BA, Ten S, Convit A (2010) Preliminary evidence for brain complications in obese adolescents with type 2 diabetes mellitus. Diabetologia 53:2298-2306

Zhang J, Wang Y, Wang J, Zhou X, Shu N, Wang Y, Zhang Z (2014) White matter integrity disruptions associated with cognitive impairments in type 2 diabetic patients. Diabetes 63:3596-3605 